\title{
Responsive robot gaze to interaction partner
}

\author{
Yuichiro Yoshikawa*, Kazuhiko Shinozawa*, Hiroshi Ishiguro*†, Norihiro Hagita* and Takanori Miyamoto* \\ * Intelligent Robotics and Communication Laboratories \\ Advanced Telecommunication Research Institute International \\ Keihanna Science City, Kyoto, 619-0288 Japan \\ Email: yoshikawa@jeap.org, \{shino, hagita, t-miyamo\}@atr.jp \\ $\dagger$ Dept. of Adaptive Machine Systems, \\ Graduate School of Engineering, Osaka University \\ 2-1 Yamadaoka, Suita, Osaka, 565-0871 Japan \\ Email: ishiguro@ams.eng.osaka-u.ac.jp
}

\begin{abstract}
Eyes play a central role in human-human communication, for example, in directing attention and regulating turntaking. For this reason, the eyes have been a central topic in several fields of interaction study. Although many psychological findings have encouraged previous work in both human-computer and human-robot interaction studies, there have been few explorations from the viewpoint of the timing of gaze behavior. In this study, the impression a person forms from an interaction is regarded to be strongly influenced by the feeling of being looked at which is assumed to be based on the responsiveness of the other's gaze to the person's one and be the basis of impression conveyance as a communicative being. In this paper, we built a robot that could move its gaze responsively to its interaction partner's one to explore the effect of responsive gaze. In this paper, we evaluated two primitive ways of controlling a robot's gaze responsively to its partner and confirmed that robots with such responsive gaze could give stronger feeling of being looked at than ones with non-responsive gaze.
\end{abstract}

\section{INTRODUCTION}

A person's gaze plays several important roles in face-toface communication, such as exhibiting one's attention and regulating turn-taking during conversation, and therefore it has been a central topic in psychology [1]. How one person looks at another seems to largely affect what kind of impression the one makes on the other. For example, people's impressions of others are known to be affected by the duration of being looked at [2], the other person's direction of gaze during face-to-face interactions [3], and so on. These facts imply the importance and the effectivity of utilizing the gaze of robots or on-screen agents for communication with humans, and therefore, have encouraged many researchers to study natural, informative, and communicative gaze for them (for example of studies with robots [4], [5], [6], [7], [8], [9], [10] and with on-screen agents [11], [12], [13]).

Consistently with the findings in psychology, it is reported that an on-screen agent can control the user's impression through parameters that control how it looks at them [4]. It has been suggested that in face-to-face situations, a robot's actions, such as making eye contact and following a person's gaze, play an important role in the ability to transfer knowledge [5] or convey its' internal state [7] to that person. It has been shown that gazes can be used to regulate turn-taking in conversational interaction [8], [11], [12]. In previous work, however, gazes are usually moved using fixed parameters so as to reproduce statistically similar gaze behavior with humans' one. In other words, the gaze behaviors were independent of their partner's one.

On the other hand, a human tends to move his/her gaze not independently of but responsively to the partner's gaze (ex. eye direction after-effect [14]). Such responsiveness of gaze seems to affect what kind of impression is perceived by the partner. For example, imagine that you get on a bus and occasionally establish eye contact with another passenger. If he/she suddenly averts his/her eyes from you, you would feel that he/she had been secretly watching at you. Or, you might feel at ease talking to someone if he/she responsively stares back at you. Inspired by these facts, we assume that humans recognize whether they are looked at by the other person based on how the other person's gaze responds to their gaze. Moreover, giving such a feeling of being looked at is considered to be an promising way to serve the feeling of being attended to by which humans might recognize the other person as their interaction partner, in other words, communicative being.

Unlike humans, the impression conveyance with crude gaze control of robots or on-screen agents is not sufficiently strong to express non-verbal messages such as the presence of communicative being for smooth communication, and therefore needs to be improved. In this paper, we address the issue of using robot gaze that is responsive to the communication partner for vivid face-to-face communication. We build a robot that can exhibit two primitive type of responsive robot gaze and evaluate their effects on partner's feeling of being looked at by the robot, which is considered to be the basis of impression conveyance in face-to-face communication, through comparison with non-responsive gazes. The rest of this paper is structured as follows; we first argue our hypothesis about responsive gaze, and then we describe the methods for implementing responsive and non-responsive gaze. After explaining about our experiments, we summarize this paper with some discussion. 


\section{HYPOTHESIS ON RESPONSIVE ROBOT GAZE}

Human gaze is assumed to convey one's attention, emotions, feelings, and so on [1]. Such impression conveyance with gaze is considered to be based on the feeling of being looked at. If one person is looking at another, the other clearly sees that they are being looked at. However, simply staring is not always sufficient for a robot to make someone feel they are being looked at. For example, if a person and a robot are facing each other, the robot can keep looking at the person simply by not moving, but this makes the robot seem like a fixed object. As a result, the impact of its gaze becomes weak. Therefore, we would like to address the issue how to strengthen the effects of robot's gaze.

\section{A. Effect of responding to the other's gaze}

The following four effects are regarded as being caused by the responsive robot gaze.

(1) When the robot alternates between looking at the person and looking elsewhere, that movement makes the person more aware of the direction of the robot's gaze.

(2) The fact that the robot has demonstrated its ability to look elsewhere makes the action of looking at the person seem more volitional.

(3) Responding to changes in gaze direction presupposes that the robot must be paying attention to the person, and so strengthens the feeling that the robot is looking at the person.

(4) It has been suggested that people recognize whether they are being looked at based on the correlation between their gaze and the other person's gaze [15]. Changing the robot's gaze direction in response to the person's gaze creates such a correlation, and therefore makes the person feel that they are being looked at.

As a result of above effects, the feeling of being looked at by the robot would be strengthened, and would be more effective at vivid impression conveyance with gaze.

\section{B. How to respond to the partner's gaze}

A following response and an averting response are two of the representative ways of responding to the partner's gaze. In a following response, the robot shifts its gaze so as to share its attention with its partner. This creates a feeling that the robot is imitating the person. If it keeps following gaze, he/she would feel the feeling of being looked at and might feel somehow threatened due to being imitated for so long. On the other hand, in an averting response, the robot shifts its gaze so as to avoid its partner's gaze, looking directly at them only when they looked away. Although the person would seldom establish eye contact with the robot, he/she would still feel the feeling of being looked at.

The robot is expected to be able to give its interaction partner various impression by combining such following and averting responses. Since one simple following or averting response cannot be optimal for all situations, the robot should have a mechanism to switch between them. In this paper, however, we focus on evaluating these two simple responses to confirm the effect of responsive gaze as the first step.

\section{IMPLEMENTATION OF RESPONSIVE ROBOT GAZE}

In this section, we introduce the control methods for robot gaze. Two of them are responsive to the partner's gaze while the remaining two are non-responsive, to be used for comparison.

\section{A. Responsive gaze}

With a following response or an averting response, the robot changes the target of its visual attention depending on what its partner is looking at. Therefore, it moves its gaze in response to the partner's gaze. In this paper, detecting what a person is looking at is accomplished by using a wearable gaze detection device.

Note that, for the smoothness of the robot motion, it ignores the partner's gaze change until 0.5 seconds have passed since the robot last changed its gaze. In the analysis of the experiment, robots with these two kinds of responsive gaze behaviors are labeled as the responsive group.

1) Following gaze: The following gaze of a robot is moved so as to share its attention with its partner. A robot with following gaze looks at the partner when he/she is looking back at the robot, and it follows the other's gaze direction if he/she looks at something else. In the analysis of this experiment, robots with following gaze are labeled as following group.

2) Averting gaze: The averting gaze of a robot is moved so as to avoid its partner's gaze, looking directly at them only when they looked away. A robot with averting gaze avoids the partner's gaze if he/she looks at the robot. Meanwhile, it looks at him/her if he/she looks away at something else. In the experiment, avoiding gaze was implemented as the gaze shift from looking at the subject face to looking at either objects. In the analysis of this experiment, robots with averting gaze are labeled as the averting group.

\section{B. Non-responsive gaze}

With a independent gaze or a $100 \%$ staring gaze, the robot either changes gaze direction independently of the partner or does not change it at all. In the analysis of this experiment, robots with these behaviors are labeled as the non-responsive group to compare with the responsive one.

1) Independent gaze: The independent gaze of a robot is moved so as to be independent of the partner's attention. A robot with independent gaze changes its target to be looked at independently of the partner's target. It is implemented by switching between tracking the partner's face and tracking other objects at variable time intervals which are randomly determined. Note that it does not change its target until at least 0.5 seconds have passed since it last changed its target. In the analysis of this experiment, robots with independent gaze are labeled as the independent group. 


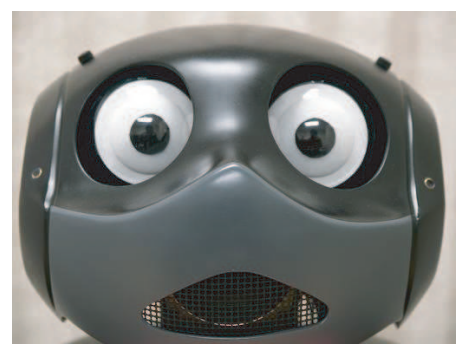

(a) Looking at a subject in front of it (b) Looking at a subject in rightside

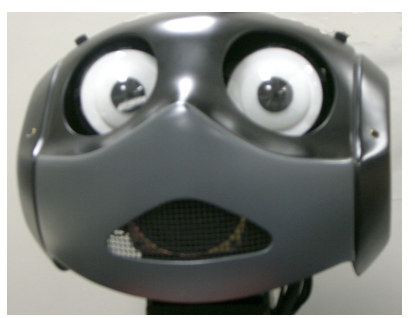

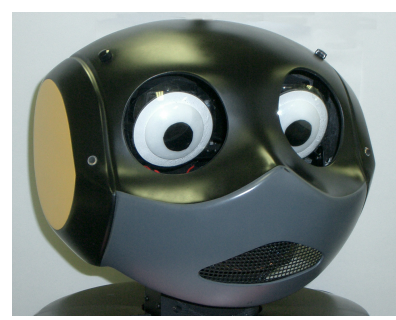

(c) Looking at the right object
Fig. 1. Samples of the robot face during looking at (a) a subject who is in front of it, (b) a subject who looks at it from right-side, and (c) the right object

2) $100 \%$ staring: The $100 \%$ staring gaze of a robot is directed so as to keep looking at the partner's face. A robot with $100 \%$ staring gaze keeps visually tracking the partner's face. The face tracking is implemented by using image processing to extract the person's face and moving the neck and eyes to keep the face in the center of the image. In the analysis of this experiment, robots with $100 \%$ staring gaze are labeled as the $100 \%$ staring group.

\section{EXPERIMENT}

To test the effect of responsive robot gaze, we hired 39 naive subjects (20 male and 19 female) and conducted an experiment with them. They were college students or graduate students whose ages ranged from 18 to 24 . The average age was 20.8 while the standard deviation was 1.5 .

\section{A. Experimental Setup}

A communication robot (Robovie-R2, ATR Robotics) was used for the experiment. Although it has 17 DOFs as a whole, only six DOFs were used, that is, the pan and tilt axes of its neck, and the pan and tilt axes of its both eyes. The unit of its eye is composed of a CMOS camera, infra-red pass film, and servo motors for eye movement. The infrared pass film is attached with the center of a white, hollow eyeball. The eyeball is covered with a transparent, semi-round acrylic resin to resemble a human eye (see Figures 1). The height of the robot was $1100[\mathrm{~mm}]$ while the horizontal and vertical size of its face was 270 [mm] and 200 [mm], respectively. The diameter of the eyes was about $70[\mathrm{~mm}]$.

The robot drives the motors of its neck and eyes based on the sensory data from its CMOS cameras in both the left and right eyes, and from a gaze detection device (EMR-8B, Nac

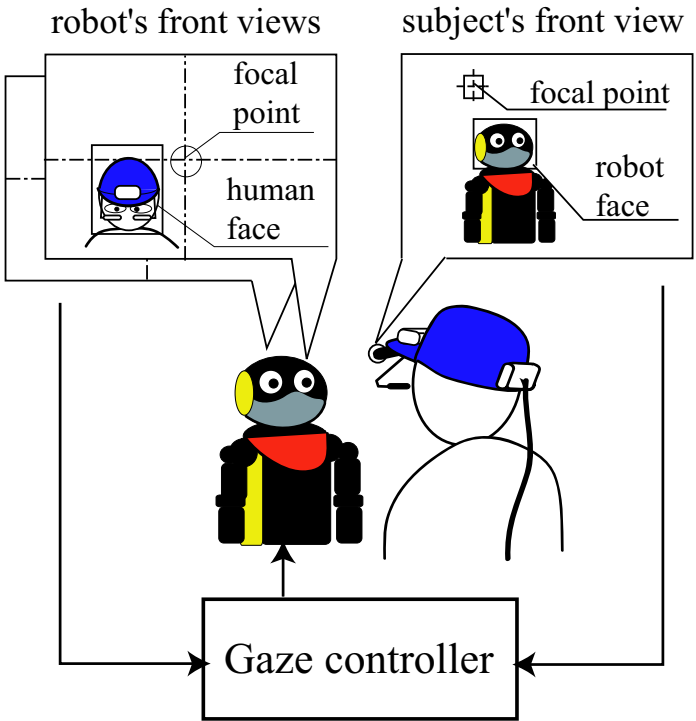

Fig. 2. The robot gaze controller which receives sensory data from its cameras and gaze data from the gaze detection device worn by a subject, and drives its servo motors to move its gaze

Image Technology Inc.) worn by the subject (see Figure 2). The gaze detection device shines infrared light on a person's eyes and records the reflection while it simultaneously captures an image of the person's frontal view with a camera mounted on the person's head. The reflected infrared image is used to calculate the person's focusing point in his/her frontal view.

The host computer (PentiumIV, $2.8 \mathrm{GHz}$ ) on the robot receives the image of the person's frontal view and the gaze data including his/her focusing point from the gaze detection device. Image processing is done for the received image to find the robot's face in the person's view. Then, it calculates whether the person is looking at the robot or at other objects based on the result of image processing and the gaze data. The host computer also receives the images from the CMOS cameras with infrared pass film in the robot's eyes. Then, it calculates the position of the person's face as the center region of infrared light reflected by the person's face.

Based on the person's gaze with respect to the robot's face, the robot determines whether to look at the person's face or at other objects. In the case of looking at the person's face, the host computer feeds the control signals to the motors of the neck so as to face with the subject and the visual feedback control signals to the motors of eyes so as to keep the partner's face in the center of the images. Figure 1(b) shows an example appearance of the robot face during looking at the face of the subject who looks at it from right-side. In the other case of looking at the objects, it feeds control signal to the motors of neck and eyes to face to other objects. The control signals to face to the other objects were pre-specified because the positions of them were fixed in this experiment. Figure 1(c) shows an example appearance of the robot face during looking at the right object. 


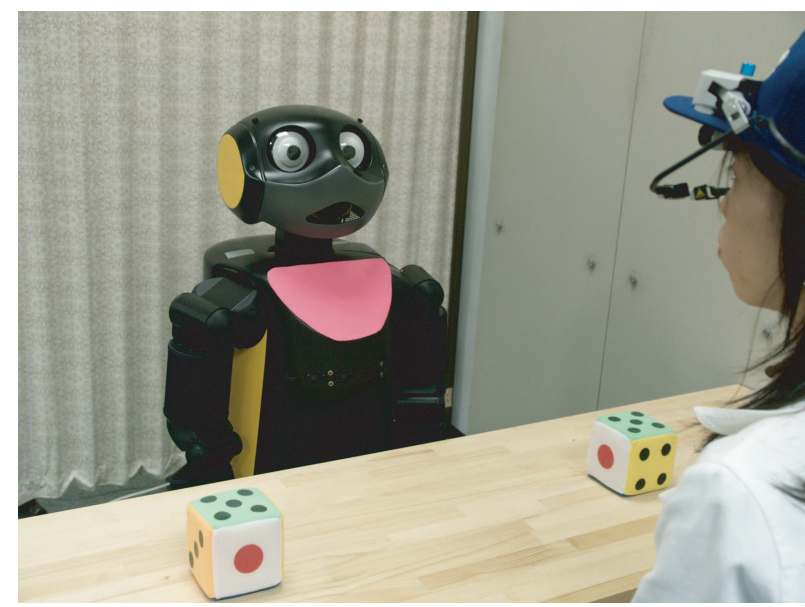

Fig. 3. An example scene from this experiment, where a subject is wearing a gaze detection device and sits across from a robot at a desk with two dice on it

\section{B. Procedure}

Before the interaction with the robot, the experimenter let a subject wear the gaze detection device and calibrated it. The experimenter did not explain that the device was used for the gaze control of the robot, but only that it was used for analysis of human-robot interaction after the experiment. Then, the subject was told that he/she would sit across from a robot that did not talk because it could not hear but could look around, and he/she would have to evaluate the robot after an interaction of about 70 seconds. To avoid having the subject keep his/her focus on the robot's face, in which case his/her gaze would not move, he/she was also instructed that when a bell was rung, he/she should look at either of two dice on the table between the subject and the robot (see Figure 3). This regulation was explained as being for the online calibration of the gaze detection device. The calibration and all instruction were done before the subject saw the robot or dice on the table.

Once the subject sat across from the robot, the robot began its gaze control. For the first eight seconds, it stared at the person's face. Then, it moved its gaze using one of four methods described earlier until 70 seconds had passed. After the 70 seconds, the subject was asked to answer the questionnaire about his/her impressions of the robot.

\section{RESUltS AND DiscusSiON}

In the experiment, subjects were asked to sit across from a robot which used one of the four gaze controlling methods listed in section III. At each session with a subject, we collected data of both the subject's and robot's gaze during interaction as well as the answers from the questionnaire. Note that each subject experienced only one of four gaze control methods. The number of the subjects for each methods is listed in Table I.
TABLE I

THE NUMBER OF SUBJECTS FOR EACH METHOD

\begin{tabular}{|c||c|c|c|}
\hline Method & Male & Female & Total \\
\hline \hline Following & 5 & 4 & 9 \\
\hline Averting & 3 & 4 & 7 \\
\hline Independent & 6 & 9 & 15 \\
\hline 100\% staringa & 6 & 2 & 8 \\
\hline
\end{tabular}

TABLE II

AVERAGE AND STANDARD DEVIATION OF TIME FOR STARING AT THE PARTNER'S FACE FOR 60 SECONDS OF INTERACTION

\begin{tabular}{|c|c|c|}
\hline Method & $\begin{array}{c}\text { Staring time by } \\
\text { the robot [sec] }\end{array}$ & $\begin{array}{c}\text { Staring time by } \\
\text { subjects [sec] }\end{array}$ \\
\hline \hline Following & $41.9 \pm 6.7$ & $45.7 \pm 5.4$ \\
\hline Averting & $18.0 \pm 7.3$ & $48.0 \pm 8.4$ \\
\hline Independent & $41.9 \pm 6.9$ & $42.2 \pm 7.9$ \\
\hline 100\% staring & $59.0 \pm 1.1$ & $47.5 \pm 11.6$ \\
\hline
\end{tabular}

\section{A. Observation of Interactions}

Figure 4 shows examples of the gaze transitions of the subject and the robot which indicate their targets of visual focus. A positive (negative) value means that the subject or the robot was focusing on the left (right) die while zero means he/she or it was focusing on the partner's face. Looking at an example from following group (see Figure 4(a)), we can see that the robot succeeded in performing a following response where it responsively looked back at the subject when he/she looked at the robot's face, and it followed the subject's gaze when looking at one of the dice. We can see an example of the interaction in averting group (see Figure 4(b)) where it responsively looked away from the subject when he/she looked at the robot's face, and it looked back when the subject looked away from the robot. In the other example from the independent group (see Figure 4(c)), we can see that it succeeded in performing independent gaze control, changing the target of focus independently of the subject's gaze. The eye movement of the robot could occur in $100 \%$ group due to the head movement of subjects. Note that the amount of eye movement in $100 \%$ staring group was measured as the standard deviation of the posture of each axis, that is 2.2 [deg] in the pan axis of right eye, $2.3[\mathrm{deg}]$ in the pan axis of left eye, and 1.0 [deg] in the tilt axis of both axes.

Table II shows the list of the average and the standard deviation of total time the robot spent looking at the subject as well as total time the subject spent looking at the robot for a 60 second period, that is, from 8 seconds to 68 seconds from the beginning of the interaction. As we can see from the list, the control parameters of the independent group were tuned so as to make the robot look at the subject for the same total amount of time as those of the following group. There is no significant difference in total time the subject spent looking at the robot between controlling methods (ANOVA, $F(3,35)=1.09, p=0.37)$.

Figure 5 shows the distribution of the average reaction time of the robot, which is defined as the average interval of time from when the subject changes his/her gaze to when the robot 


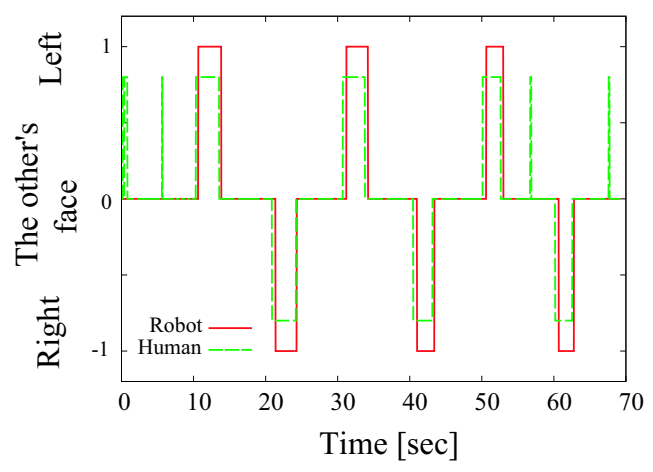

(a) Following

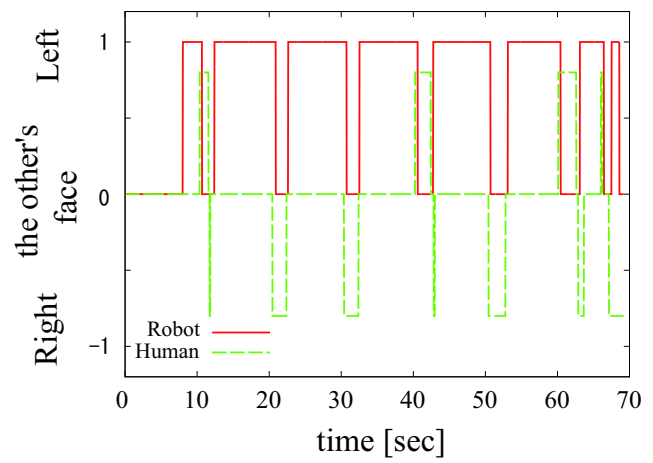

(b) Averting

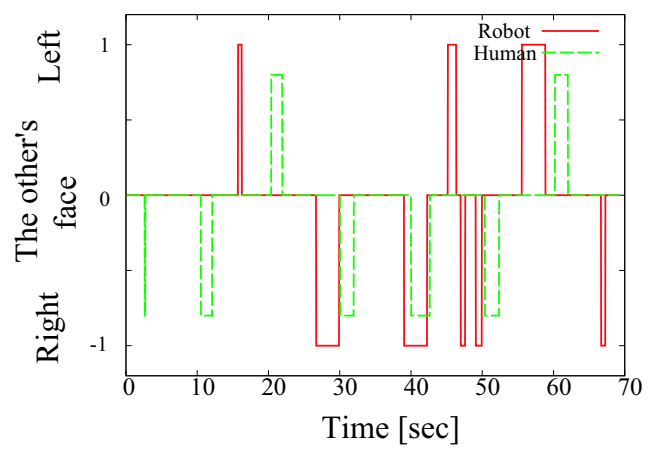

(c) Independent

Fig. 4. Examples of the transition of gaze between the subject's face and the objects on the table in (a) the following group and (b) the averting group, and (c) the independent group

changes its gaze. Note that the cases where it changed its gaze after more than five second were eliminated from the data when calculating the average because they were not regarded as responsive movement. As we can see from the graph, the robot had a more rapid response to the subject's gaze in the following and averting group compared to the independent group.

\section{B. The feeling of being looked at}

It was predicted that (1) robots that change the focus of their visual attention give their partners a stronger feeling of being looked at than robots that do not change their gaze direction, and that (2) robots with responsive gaze give their
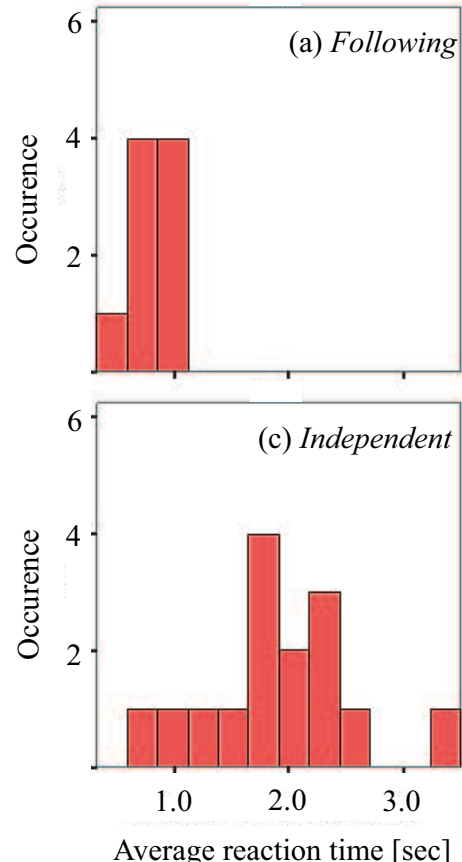

Fig. 5. Histograms of the average reaction time of the robot in each control method: (a) following, (b) averting, and (c) independent

partners a stronger feeling of being looked at than robots with independent gaze control. We conducted a statistical analysis on the average score for the question "Did you feel like the robot was looking at you?" as an indicator of the feeling of being looked at. Note that the subject answered the question on a scale of one to seven, where high value corresponds to a strong feeling of being looked at and vice versa. For such a priori comparison, we applied the process of reverse Helmert contrast for four groups, that is, the following, averting, independent, and $100 \%$ staring group, in that order.

We call a group that consists of following, averting, and independent groups the gaze-changing group. By contrasting the gaze-changing group with the $100 \%$ staring group, we confirmed that the gaze-changing group gave subjects a significantly stronger feeling of being looked at than the $100 \%$ staring group $(p=0.001)$ (see Figure 6). Therefore, it seems that subjects had a stronger feeling of being looked at by robots in the gaze-changing group than by robots in the 100\% staring group. Note that we did not see significant interaction between gender and methods of gaze control.

By contrasting the responsive group with the independent one, we saw that robots in the responsive group exhibited weak tendency of giving subjects a stronger feeling of being looked $a t$ than in the independent group $(p=0.084)$. However, the independent group includes cases where the robot occasionally moved its gaze in a way that seemed like it could have been a response to the subject. Therefore, to take such occasional responses into account, we analyzed the average response time 


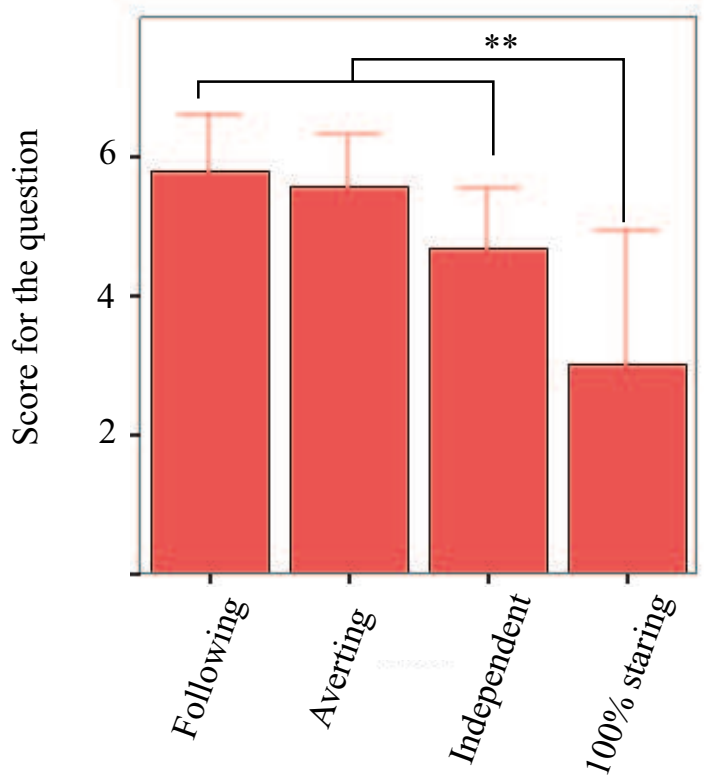

Fig. 6. Score for the question, "did you feel like the robot was looking at you?" with respect to four methods of gaze control

TABLE III

SPEARMAN'S CORRELATION COEFFICIENT BETWEEN THE SCORE FOR THE QUESTION, "DID YOU FEEL LIKE THE ROBOT WAS LOOKING AT YOU?" AND THE AVERAGE REACTION TIME TO PARTNER'S GAZE SHIFT DURING FIRST AND LAST 30 SECONDS OF INTERACTION IN THE independent GROUP

\begin{tabular}{|c||c|c|}
\hline & $\begin{array}{c}\text { reaction time } \\
\text { in fi rst } 30 \mathrm{sec}\end{array}$ & $\begin{array}{c}\text { reaction time } \\
\text { in last } 30 \mathrm{sec}\end{array}$ \\
\hline \hline correlation coeffi cient & -0.69 *** $\left.^{*}\right)$ & -0.31 \\
\hline signifi cance probability & 0.004 & 0.256 \\
\hline $\mathrm{N}$ & 15 & 15 \\
\hline
\end{tabular}

of the robots in the independent group. As we can see from Figure 7 and Table III, there was negative correlation between the average response time during the first 30 seconds and the degree of feeling of being looked at in the independent group.

Therefore, for further analysis, we divided the independent group into an involuntary responsive group and an true independent group based on the average reaction time. The average reaction time in the responsive group ranged between $0.30 \sim 1.33$ seconds. Therefore, the involuntary responsive group was defined as consisting of the cases in which the average reaction time was less than 1.33 seconds while the true independent one was defined as consisting of remaining cases in the independent one. Then, we define a group that consists of the responsive and involuntary responsive groups, which we call the extended responsive group, and contrasted it with the true independent one. We confirmed that subjects had stronger feeling of being looked at for robots in the extended responsive group than for robots in the true independent group $(p=0.009)$ (see Figure 8). Therefore, regardless of the nature of the response, the robot succeeded in giving subjects

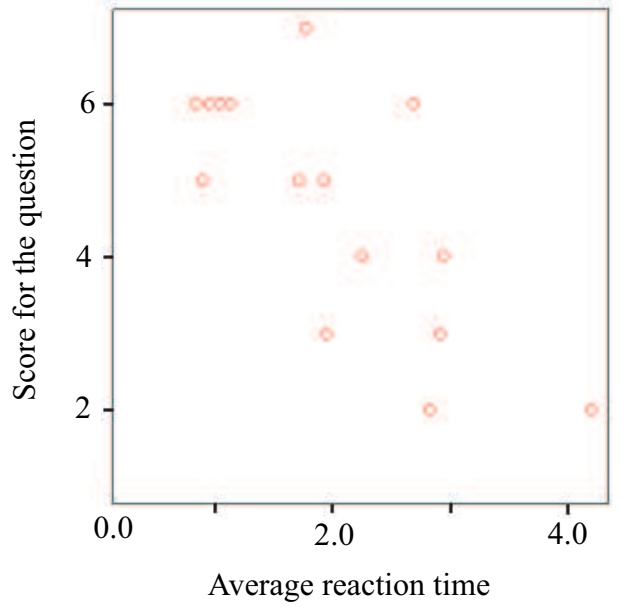

Fig. 7. Score for the question, "did you feel like the robot was looking at you?" with respect to the average reaction time to subjects' gaze shift in fi rst 30 seconds of interaction

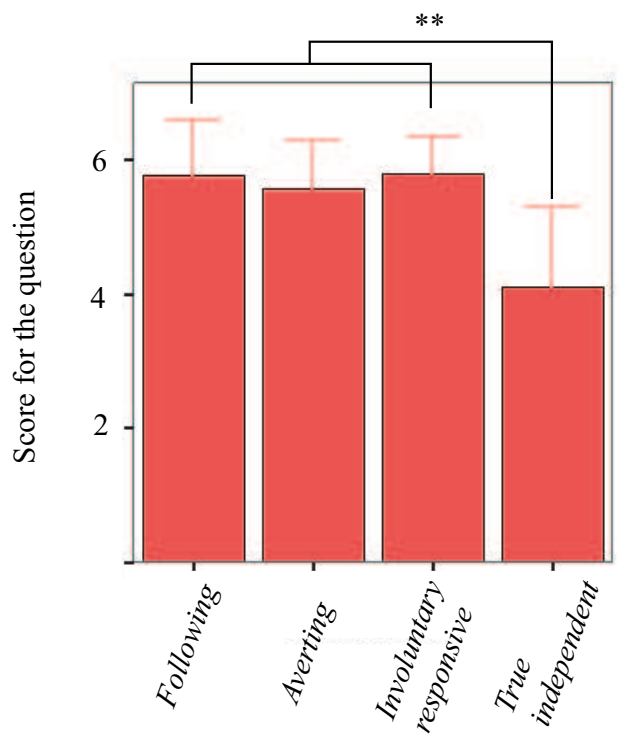

Fig. 8. Score for the question, "did you feel like the robot was looking at you?" with respect to the modifi ed conditions

a stronger feeling of being looked at by responding to the subject's gaze.

In addition, there did not seem to be a correlation between the average reaction time in the last 30 seconds and the score for feeling of being looked at for the independent group (see Table III). Therefore, to create a stronger feeling of being looked at, it seems to be important that a robot shows gaze control responsive to the subject's gaze from the beginning of interaction. Implementation of independent gaze is easy since it does not require obtaining information about the partner's gaze. However, it is not guaranteed that a robot with an independent controller will always show a responsive behavior. 
A responsive gaze controller, such as our proposed method, is expected to show gaze control responsive to the subject's gaze from the beginning, and therefore elicit a stronger feeling of being looked at.

\section{CONCLUSION}

In this paper, we proposed two methods of robot gaze control that are responsive to the partner's gaze and evaluated their effects on the partner's impression through an experiment with 39 subjects. From the statistical analysis, we confirmed that robots with responsive gaze control succeeded in giving subjects a stronger feeling of being looked at than those with non-responsive gaze control. We expect that such a feeling would be a basis for conveying vivid impression using gaze. Since the suitable gaze response to the partner is considered to depend on what kind control of impression to be conveyed and the context of interaction, future work should address ways to integrate the proposed methods of responsive gaze control. Furthermore, the behavior of gaze and one's impression of it depend on cultural and personal factors. Therefore, a robot needs to adapt its ways of gaze control to its partner's characteristics, which is also an important issue to address in the future.

\section{ACKNOWLEDGMENT}

This research was supported by the Ministry of Internal Affairs and Communication.

\section{REFERENCES}

[1] C. Kleinke, Gaze and Eye Contact: A Research Review, Psychological Bulletin, vol. 100, no. 1, pp. 78-100, 1986.

[2] M. Cook and M. C. Smith, The Role of Gaze in Impression Formation, British Journal of Social Clinical Psychology, vol. 14, pp. 19-25, 1975D

[3] J. W. Tankard Jr., Effects of eye position on person perception, Perceptual \& Motor Skills, vol. 31, pp. 883-893, 1970.

[4] A. Fukayama, T. Ohno,N. Mukawa, M. Sawaki, and N. Hagita, Messages Embedded in Gaze of Interface Agents -Impression management with agent's gaze -, In Proceedings of the ACM Conference on Human Factors in Computing Systems, CHI2002, vol. 1, pp. 41-49, 2002.

[5] K. Shinozawa, F. Naya, K. Kogure, and J. Yamato, Effect of robot's tracking users on human decision making, In Proceedings of the 2004 IEEE/RSJ International Conference on Intelligent Robots and Systems, pp. 1908-1913, 2004.

[6] C. Breazeal, A. Edsinger, P. Fitzpatrick, and B. Scassellati, Active Vision for Sociable Robots, IEEE Transactions on Systems, Man, and Cybernetics -Part A: Systems and Humans, vol. 31, No. 5, pp. 443-453, 2001.

[7] C. Breazeal, C. Kidd, A. L. Thomaz, G. Hoffman, and M. Berlin, Effects of Nonverbal Communication on Effi ciency and Robustness in HumanRobot Teamwork, In Proceedings of the 2005 IEEE/RSJ International Conference on Intelligent Robots and Systems, 2005.

[8] C. L. Sidner, C. D. Kidd, C. H. Lee, and N. Lesh, Where to look: A study of human-robot engagement, In Proceedings of ACM International Conference on Intelligent User Interfaces, pp. 78-84, 2004.

[9] H. Sumioka, K. Hosoda, Y. Yoshikawa, and M. Asada, Motion-triggered human-robot synchronization for autonomous acquisition of joint attention, In Proceedings of the 4th IEEE International Conference on Development and Learning, 2005.

[10] A. Ito, S. Hayakawa, and K. Terada, Why Robots Need Body for Mind Communication - An Attempt Of Eye-Contact between Human and Robot -, In Proceedings the 2004 IEEE International Workshop on Robot and Human Interactive Communication, pp. 473-478, 2004.

[11] M. Garau, M. Slater, S. Bee, and M. A. Sasse, The impact of Eye Gaze on Communication using Humanoid Avators, In Proceedings of the SIGCHI conference on Human factors in computing systems, pp. 309-316 2001.
[12] D. Heylen, I. van Es, A. Nijholt, and B. van Dijk, Experimenting with the Gaze of a Conversational Agent, Intelligent and Effective Interaction in Multimodal Dialogue System, Kuppevelt et al.(eds.), Kluwer Academic Publishers, 2003.

[13] Cassell and Vilhjalmsson, Fully Embodied Conversational Avators: Making Communicative Behaviors Autonomous, Autonomous Agents and Multi-Agent Systems, vol. 2, pp. 45-64, 1999.

[14] J. Seyama, and R. S. Nagayama, Eye direction aftereffect, Psychological Research, vol. 70, No.1, pp. 59-67, 2006.

[15] A. Ito, An approach to the eye contact through the outstaring game niramekkoCIn Procedings of 11th IEEE International Workshop on Robot and Human Interactive Commnication, ROMAN2002, pp. 405-410, 2002. 